\title{
Estabilidad y control de sistemas mecánicos de base móvil
}

\author{
D. A. Bravo M. \\ Ingeniero Físico, PhD. Profesor Departamento de Física. Universidad del Cauca, \\ Calle 5 No. 4-70, Popayán, Colombia. \\ email:dibravo@unicauca.edu.co \\ C. F. Rengifo R. \\ Ingeniero Electricista, PhD. Profesor Departamento de Electrónica, Instrumentación y Control, \\ Universidad del Cauca, Calle 5 No. 4-70, Popayán, Colombia. \\ email: caferen@unicauca.edu.co
}

Received 24 June 2020; accepted 7 August 2020

\begin{abstract}
En este trabajo se estudia la estabilidad de un péndulo invertido de dos grados de libertad y de base móvil, el cual se modeló mediante la formulación Euler-Lagrange. Este modelo permitió diseñar e implementar una estrategia de control para el seguimiento de trayectorias articulares de referencia. Los sistemas mecánicos de bases fijas y móviles permiten ilustrar un concepto fundamental en las ciencias físicas, que es la diferencia entre limitaciones tecnológicas y limitaciones fundamentales.

Descriptores: Enseñanza; ecuación de Lagrange; sistemas mecánicos; simulación.

In this paper, we study the stability of an inverted pendulum with two degrees of freedom and a mobile base, which was modeled using the Euler-Lagrange formulation. This model is used to design and implement a control strategy for tracking joint reference trajectories. The mechanical systems of fixed and mobile base illustrate a fundamental concept in the physical sciences, which is the difference between technological and fundamental limitations.
\end{abstract}

Keywords: Equation; dynamical systems; simulation.

PACS: 01.40.Fk; 01.40.Ha; 01.50.H-

DOI: https://doi.org/10.31349/RevMexFisE.18.69

\section{Introducción}

Los sistemas mecánicos se pueden clasificar como de base fija o de base móvil. Los primeros, como los brazos manipuladores industriales, tienen una base inmóvil con respecto al suelo que soporta las aceleraciones de cada una de sus articulaciones, sin importar la trayectoria de referencia. En contraposición, los de base móvil, como su nombre lo indica, no están fijos con respecto al suelo. Un ejemplo son los robots bípedos, donde las aceleraciones de las articulaciones de las piernas pueden hacer que el robot pierda estabilidad y caiga $[1,2]$.

Un sistema mecánico de base móvil de gran interés para ilustrar varios conceptos en física e ingeniería de control es el péndulo invertido, el cual comparte las mismas características de una bicicleta, [3]. Tales particularidades han incrementado el interés de muchos investigadores en proporcionar herramientas de enseñanza e investigación que permitan afianzar los conocimientos relacionados con las diferentes estrategias de control y estabilidad de la base mientras se controla la posición del brazo del péndulo para una trayectoria asignada.

En este trabajo se modela y controla un péndulo invertido dos grados de libertad (GDL) cuya base no está fija al suelo (ver Fig. 1). El objetivo de control es que las posiciones angulares $q_{1}$ y $q_{2}$ obedezcan unas consignas preestablecidas $\left(q_{1}^{d}(t)\right.$ y $\left.q_{2}^{d}(t)\right)$. Adicionalmente, el control debe garantizar, en medida de lo posible, que el movimiento de los brazos del péndulo no conlleve al deslizamiento o a la inestabilidad de la base, sobre la cual el suelo ejerce fuerzas de reacción (normal y tangencial). Sin embargo, es importante precisar que para ciertas trayectorias de referencia no puede existir un controlador que satisfaga este requerimiento. Este es el caso, por ejemplo, de las consignas articulares $q_{1}^{d}(t) \equiv 90^{\circ}$ y $q_{2}^{d}(t) \equiv 0^{\circ}$, que cuando las masas de los dos brazos superan a la de la base, el sistema

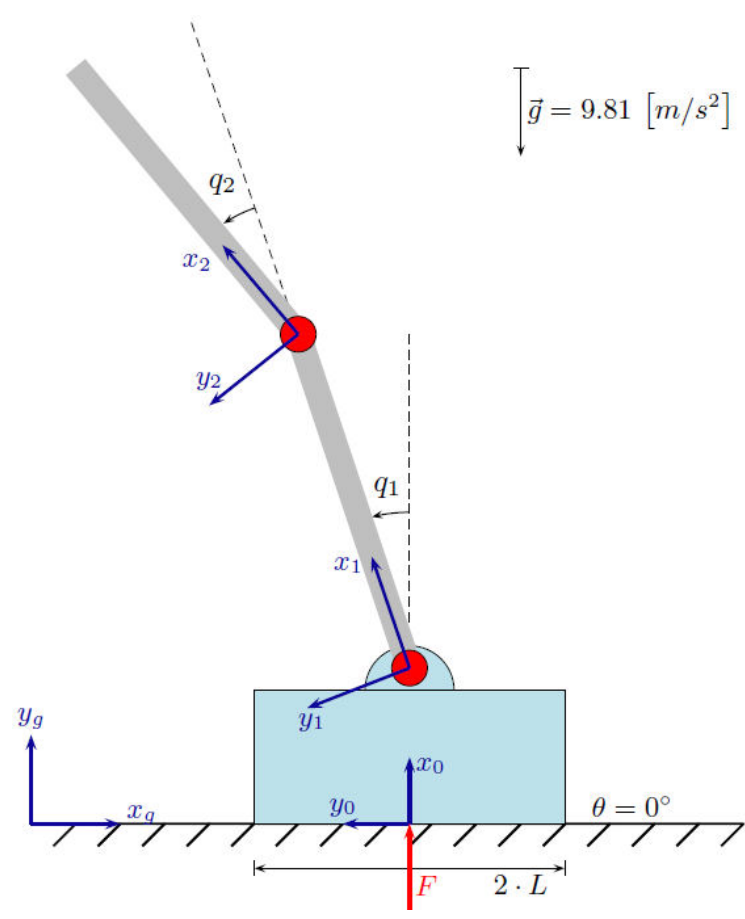

FIgURA 1. Péndulo Invertido de 2 GDL. 
se volcará hacia el lado izquierdo. A esta característica se le conocerá como una limitación fundamental del sistema. Cuando esta limitación no está presente, el controlador debe ser robusto ante disturbios externos, dinámicas no modeladas, errores en la estimación de los parámetros y ruido en las medidas, entre otros [5].

En los sistemas mecánicos de base fija, como los brazos de robot utilizados en la industria de manufactura, el seguimiento de trayectorias articulares está limitado únicamente por la capacidad de los actuadores y la resistencia de los componentes mecánicos. En contraposición, en los sistemas de base móvil existen limitaciones fundamentales en cuanto a las consignas que se pueden imponer a las articulaciones. Cuando la base es móvil, se debe garantizar que las aceleraciones articulares no inducirán fuerzas de reacción que conlleven la inestabilidad de la base del mecanismo. Este problema se ha abordado desde dos perspectivas. La primera consiste en concebir movimientos articulares basados en técnicas de optimización con restricciones, como las presentadas en $[6,7,2]$, que aseguran la inmovilidad de la base y el no deslizamiento de esta con respecto al suelo. $\mathrm{Su}$ principal dificultad es que requieren de un conocimiento preciso del modelo matemático del sistema. La segunda perspectiva, propuesta en [8] bajo el nombre de Time Scaling, se fundamenta en la utilización de estrategias de control orientadas al seguimiento geométrico pero no temporal de las consignas articulares. Esto significa que el controlador garantiza que la secuencia de posiciones angulares de las articulaciones será igual a la de referencia, pero que los tiempos en que se alcanzarán dichas posiciones diferirán de los deseados. Cabe resaltar que el seguimiento geométrico de trayectorias aún está sujeto a limitaciones fundamentales. Considere, por ejemplo, que en el sistema de la Fig. 1 la longitud de cada péndulo es de $50 \mathrm{~cm}$, el ancho de la base es $10 \mathrm{~cm}$ y que las masas de los péndulos son 10 veces mayores que la masa de la base. En tal escenario, será imposible seguir una trayectoria donde $q_{1}$ vaya $-90^{\circ}$ a $90^{\circ}$ grados. Así, los sistemas mecánicos de bases fijas y móviles permiten ilustrar un concepto fundamental en las ciencias físicas, que es la diferencia entre limitaciones tecnológicas y limitaciones fundamentales.

En la revisión del estado del arte consultado se encuentra la aplicación de distintas estrategias de control para estos sistemas, tanto lineales como no lineales. Ambas técnicas requieren de una buena comprensión del modelo matemático [9] que puede obtenerse mediante la ecuación de Lagrange [10], el algoritmo de Newton-Euler [11], leyes de Newton [12]. Una comparación entre estrategias de control lineales y no lineales se encuentran en [13-15].

El artículo se estructura de la siguiente manera: la Sec. 2 describe el modelo matemático del péndulo invertido de dos GDL y la dinámica del punto de momento cero, usado para definir la estabilidad de sistemas mecánicos de base móvil, la Sec. 3 está dedicada a la estrategia de control, seguida por los resultados en la $\mathrm{Sec}$. 4 y finalizando con las conclusiones del artículo en la Sec. 5.

\section{Modelo dinámico}

Para modelar el sistema mecánico se siguieron las recomendaciones presentadas en [16]. La representación Lagrangiana del modelo dinámico directo en tiempo continuo del péndulo invertido de dos grados de libertad y base móvil, ver Fig. 1, es el siguiente:

$$
\mathbf{A}(q) \ddot{q}+H(q, \dot{q})=\mathbf{B} \Gamma+\mathbf{J}^{\mathrm{T}}(q) F
$$

donde $q \in \mathbb{R}^{5}$ y $\dot{q} \in \mathbb{R}^{5}$ con los vectores de posiciones y de velocidades generalizadas, de manera que

$$
q \triangleq\left[\begin{array}{c}
{ }^{\mathrm{g}} x_{0} \\
{ }^{\mathrm{g}} y_{0} \\
\theta \\
q_{1} \\
q_{2}
\end{array}\right] \quad \dot{q} \triangleq\left[\begin{array}{c}
\mathrm{g} \dot{x}_{0} \\
{ }^{\mathrm{g}} \dot{y}_{0} \\
\dot{\theta} \\
\dot{q}_{1} \\
\dot{q}_{2}
\end{array}\right]
$$

El vector $q$ contiene las 2 posiciones articulares $\left(q_{1}, q_{2}\right)$, la posición y la orientación del sistema de referencia < $x_{0}, y_{0}>$ con respecto al referente $<x_{g}, y_{g}>$. La matriz $\mathbf{A}(q) \in \mathbb{R}^{5 \times 5}$ es la matriz de inercia. El vector $H(q, \dot{q}) \in$ $\mathbb{R}^{5}$ contiene el efecto de las fuerzas de gravedad, centrífugas y de Coriolis. El vector $\Gamma \in \mathbb{R}^{2}$ contiene el par motor de cada una de las 2 articulaciones accionadas en el péndulo. La matriz $\mathbf{B} \in \mathbb{R}^{5 \times 2}$ está definida de tal manera que, al multiplicarla por el vector $\Gamma$, se obtiene un vector cuyas 3 primeras componentes son cero, y las 2 siguientes son las componentes de $\Gamma$. La matriz $\mathbf{B}$ indica que las 3 primeras coordenadas (posición y orientación absolutas) no son accionadas directamente.

$\mathbf{J} \in \mathbb{R}^{3 \times 5}$ permite expresar las velocidades lineal y angular de la base móvil del péndulo con respecto al suelo. El vector $F \in \mathbb{R}^{3}$ contiene las fuerzas y el momento de reacción generados por el contacto entre la base y el suelo.

Las incógnitas del modelo anterior son $\ddot{q} \in \mathbb{R}^{5}$ y $F \in$ $\mathbb{R}^{3}$. Es decir, hay un total de 5 ecuaciones y 8 incógnitas. El modelo anterior, sin embargo, está sujeto a las restricciones de inmovilidad de la base del péndulo:

$$
\left[\begin{array}{c}
{ }^{\mathrm{g}} p_{\mathrm{x}}(q) \\
{ }^{\mathrm{g}} p_{\mathrm{y}}(q) \\
\theta(q)
\end{array}\right] \equiv 0
$$

Con el propósito de involucrar las incógnitas del problema en las tres ecuaciones anteriores, estas se derivan dos veces con respecto al tiempo. Al derivar una vez se obtiene la Ec. (4).

$$
\left[\begin{array}{c}
{ }^{\mathrm{g}} \dot{p}_{\mathrm{x}} \\
{ }^{\mathrm{g}} \dot{p}_{\mathrm{y}} \\
\dot{\theta}
\end{array}\right]=\mathbf{J}(q) \dot{q} \equiv 0
$$

Al derivar una vez más, las restricciones conllevan a ecuaciones que dependen de $\ddot{q}$

$$
\left[\begin{array}{c}
\mathrm{g} \ddot{p}_{\mathrm{x}} \\
{ }^{\mathrm{g}} \ddot{p}_{\mathrm{y}} \\
\ddot{\theta}
\end{array}\right]=\mathbf{J}(q) \ddot{q}+\dot{\mathbf{J}}(q) \dot{q} \equiv 0 .
$$


Al combinar la Ecs. (1) y (5) se obtiene el modelo dinámico directo del péndulo. Este permite calcular $\ddot{q}$ y $F$ en función de $q, \dot{q}$ y $\Gamma$.

$$
\left[\begin{array}{cc}
\mathbf{A}(q) & -\mathbf{J}^{\mathrm{T}}(q) \\
\mathbf{J}(q) & \mathbf{0}
\end{array}\right]\left[\begin{array}{l}
\ddot{q} \\
F
\end{array}\right]=\left[\begin{array}{c}
\mathbf{B} \Gamma-H(q, \dot{q}) \\
-\mathbf{J}(q) \dot{q}
\end{array}\right]
$$

Al reescribir la restricción, la Ec. (5) se expresaría de la siguiente manera:

$$
\mathbf{J}_{u}(q) \ddot{q}_{u}+\mathbf{J}_{a}(q) \ddot{q}_{a}+\dot{\mathbf{J}}(q) \dot{q}=0,
$$

donde $q_{u}$ es el vector de las coordenadas no-accionadas (pasivas) y $q_{a}$ es el vector de las coordenadas accionadas (activas). Con ello, se resuelve $\ddot{q}_{u}$ de la Ec. (7).

$$
\ddot{q}=\left[\begin{array}{l}
\ddot{q}_{u} \\
\ddot{q}_{a}
\end{array}\right]=\left[\begin{array}{c}
-\mathbf{J}_{u}^{-1}(q) \mathbf{J}_{a}(q) \\
\mathbf{I}
\end{array}\right] \ddot{q}_{a}+\left[\begin{array}{c}
-\mathbf{J}_{u}^{-1}(q) \dot{\mathbf{J}}(q) \dot{q} \\
\mathbf{0}
\end{array}\right] .
$$

La ecuación anterior indica que el contacto entre la base y el suelo conlleva a que las aceleraciones de las coordenadas no-accionadas dependan directamente de las aceleraciones de las coordenadas accionadas. La Ec. (8) describe un sistema autónomo variante en el tiempo, de orden inferior al modelo Ec. (1) y cuya estabilidad depende directamente de la trayectoria de referencia. Para aligerar la notación matemática se definen las siguientes variables:

$$
\begin{gathered}
\mathbf{M}(q) \triangleq\left[\begin{array}{c}
-\mathbf{J}_{u}^{-1}(q) \mathbf{J}_{a}(q) \\
\mathbf{I}
\end{array}\right] \\
\mathbf{N}(q, \dot{q}) \triangleq\left[\begin{array}{c}
-\mathbf{J}_{u}^{-1}(q) \dot{\mathbf{J}}(q) \dot{q} \\
\mathbf{0}
\end{array}\right] .
\end{gathered}
$$

Al sustituir $\ddot{q}=\mathbf{M} \ddot{q}_{a}+\mathbf{N}$ en el modelo dinámico Ec. (1) se tiene:

$$
\begin{aligned}
\mathbf{A}(q) \mathbf{M}(q) \ddot{q}_{a} & +\mathbf{A}(q) \mathbf{N}(q, \dot{q})+H(q, \dot{q}) \\
& =\mathbf{B} \Gamma+\mathbf{J}^{\mathrm{T}}(q) F
\end{aligned}
$$

Al premultiplicar la Ec. (9) por $\mathbf{M}^{T}(q)$ y simplificarla se obtiene:

$$
\begin{aligned}
\Gamma & =\mathbf{M}^{\mathrm{T}}(q) \mathbf{A}(q) \mathbf{M}(q) \ddot{q}_{a} \\
& +\mathbf{M}^{\mathrm{T}}(q) \mathbf{A}(q) \mathbf{N}(q, \dot{q})+\mathbf{M}^{\mathrm{T}}(q) H(q, \dot{q}) .
\end{aligned}
$$

Si $\ddot{q}_{a}$ se remplaza por la aceleración deseada $\ddot{q}_{a}^{d}$, la Ec. (10) se convierte en un control por par calculado CTC (Computed Torque Control), [17].

$$
\ddot{q}_{a}^{d}=\ddot{q}^{r}+k_{v}\left(\dot{q}^{r}-\dot{q}_{a}\right)+k_{p}\left(q^{r}-q_{a}\right),
$$

donde $q_{a}^{r} \in \mathbb{R}^{2}$ y $\ddot{q}_{a}^{r} \in \mathbb{R}^{2}$ y $\ddot{q}_{a}^{r} \in \mathbb{R}^{2}$ son las trayectorias de referencia deseadas para las articulaciones $q_{1}$ y $q_{2}$. Al reemplazar $\ddot{q}_{a}^{d}$ en la Ec. (10) se obtienen las siguientes ecuaciones que permiten calcular el vector de par motor $\Gamma$ requerido por las coordenadas articulares $\left(q_{1}, q_{2}\right)$ para seguir las trayectorias de referencia $q_{a}^{d}$.

$$
\begin{aligned}
\Gamma & =\mathbf{M}^{\mathrm{T}} \mathbf{A} \mathbf{M} \ddot{q}_{a}^{d}+\mathbf{M}^{\mathrm{T}} \mathbf{A} \mathbf{N}+\mathbf{M}^{\mathrm{T}} H \\
\ddot{q}_{a}^{d} & =\ddot{q}^{r}+k_{v}\left(\dot{q}^{r}-\dot{q}_{a}\right)+k_{p}\left(q^{r}-q_{a}\right)
\end{aligned}
$$

La ley de control (12) asegura que las articulaciones accionadas siguen a las referencias deseadas. Sin embargo, cuando esto sucede, las articulaciones pasivas dependen directamente de las accionadas; a su vez, la estabilidad del sistema dinámico depende directamente de las trayectorias de referencia.

\subsection{Dinámica del punto de momento cero}

Vukobratović [18] introdujo por primera vez el concepto de punto de momento cero (ZMP), el cual se utiliza para el control de la marcha en robots humanoides. Este especifica el punto donde las fuerzas de reacción generadas por el contacto del pie con el suelo no producen ningún momento en el plano de contacto. El ZMP supone que la superficie de contacto es plana y que tiene un coeficiente de fricción suficiente para evitar el deslizamiento.

En este trabajo se extiende este concepto para la estabilidad de sistemas mecánicos, entendiendo la estabilidad como la propiedad de un cuerpo rígido para mantenerse inmóvil sobre cada uno de sus ejes de rotación en un marco de referencia tridimensional. Si las fuerzas de contacto entre un objeto y el suelo conllevan a una fuerza y a un momento resultantes $F_{0} \in \mathbb{R}^{3}$ y $M_{0} \in \mathbb{R}^{3}$, respectivamente, entonces el momento $M_{R} \in \mathbb{R}^{3}$ alrededor de un punto $\vec{R} \in \mathbb{R}^{3}$ estará dado por:

$$
M_{R}=M_{0}-\vec{R} \times F_{0},
$$

donde $F_{0}, M_{0}$ y $\vec{R}$ se suponen expresados en el sistema coordenado $<x_{0}, y_{0}, z_{0}>$ de la Fig. 2. Si $M_{R}$ es cero y adicionalmente $\vec{R}$ está sobre el plano de contacto entre el objeto y el suelo (representado en color azul en la Fig. 2), entonces $\vec{R}$ se denomina como el punto de momento cero o ZMP. Para el caso bidimensional, el ZMP se calcula a partir de (13), así:

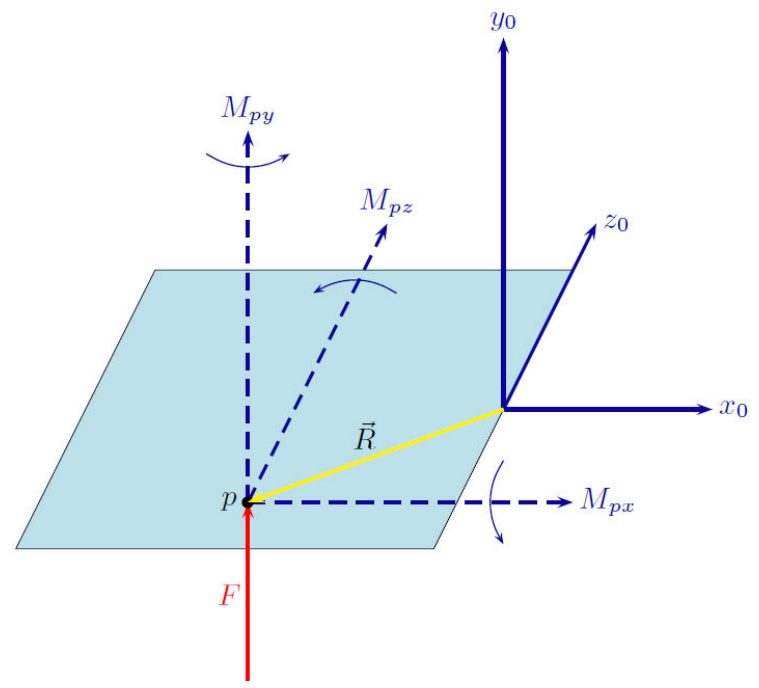

FIGURA 2. Momentos generados en un punto $p$ sobre el polígono de sustentación. 


$$
\left[\begin{array}{c}
0 \\
0 \\
m_{z}
\end{array}\right]-\left[\begin{array}{c}
p_{x} \\
0 \\
0
\end{array}\right] \times\left[\begin{array}{c}
f_{x} \\
f_{y} \\
0
\end{array}\right]=\left[\begin{array}{l}
0 \\
0 \\
0
\end{array}\right] .
$$

La ecuación anterior se convierte en:

$$
m_{z}-f_{y} \cdot p_{x}=0
$$

donde $p_{x}$ es el punto de momento cero, $m_{z}$ es el momento angular en la dirección del eje $z$ y $f_{y}$ es la fuerza normal de reacción del suelo. El sistema dinámico se mantendrá estable si $p_{x}$ se encuentra dentro del polígono de soporte. Esto significa que si el origen de $\left\langle x_{0}, y_{0}\right\rangle$ se encuentra en el punto medio de la base del objeto en contacto con el suelo y la longitud de la base es $2 L$, entonces se debe satisfacer:

$$
-L \leq p_{x} \leq L
$$

\section{Control de Sistemas Mecánicos de Base Móvil}

El objetivo de la estrategia de control presentada en esta sección es el seguimiento de trayectorias parametrizadas mediante splines cúbicos.

\subsection{Conjunto de trayectorias de referencia}

La trayectoria para la articulación $i(i=1,2)$ se representa mediante un conjunto de $N$ polinomios de tercer orden llamados splines cúbicos, [19].

$$
q_{i}^{r}(t)=c_{0, j, i}+c_{1, j, i} t+c_{2, j, i} t^{2}+c_{3, j, i} t^{3}
$$

$c_{0, j, i}, c_{1, j, i}, c_{2, j, i}, c_{3, j, i}$ son los coeficientes de un polinomio de tercer orden y cada polinomio representa la trayectoria de referencia de la articulación $i$ en el intervalo de tiempo $t \in\left[t_{j-1}, t_{j}\right](j=1 \ldots N+1)$. La longitud de cada intervalo de tiempo es constante $\left(h=t_{j}-t_{j-1}\right)$. Por ejemplo,
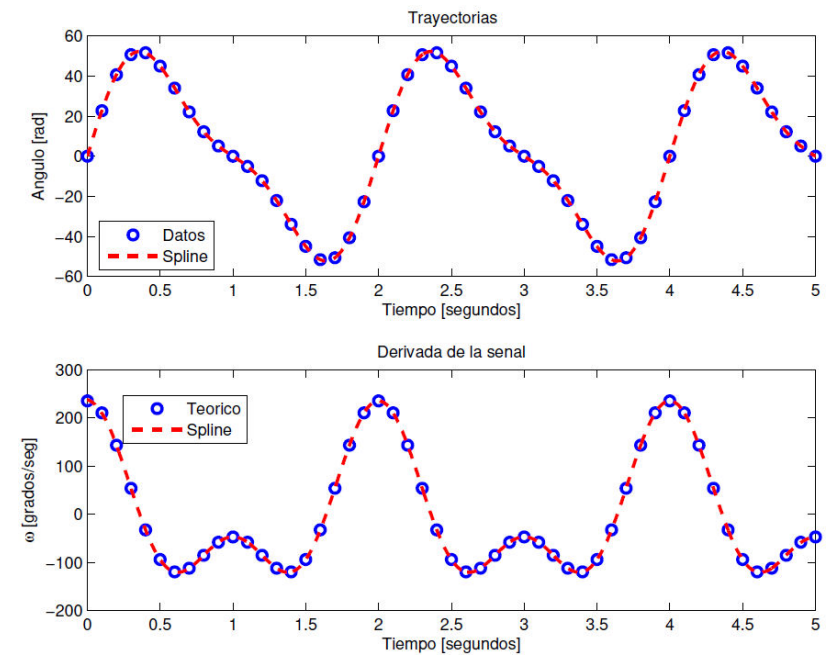

FIGURA 3. Interpolación basada en spline cúbicas. la Fig. 3 presenta la aproximación mediante splines cúbicos de la señal $q_{1}^{r}(t)=45 \sin \omega t+15 \sin \omega t$ y de su derivada $\dot{q}_{1}^{r}(t)=45 \omega \cos \omega t+15 \omega \cos \omega t$, para $\omega=\pi$ y $h=0.1$ segundos. Si se considera que el intervalo de simulación es de 5 s y que $h=0.1$, entonces el número de splines utilizadas para la aproximación fue $N=50$.

\section{Resultados y discusión}

Se diseñó un experimento para observar la estabilidad del sistema dinámico a partir de dos trayectorias de referencia. Las condiciones iniciales para la simulación son:

$$
q(0)=\left[\begin{array}{c}
{ }^{\mathrm{g}} x_{0}(0) \\
{ }^{\mathrm{g}} y_{0}(0) \\
\theta(0) \\
q_{1}(0) \\
q_{2}(0)
\end{array}\right]=\left[\begin{array}{c}
0 \\
0 \\
90^{\circ} \\
0 \\
0
\end{array}\right] \quad \dot{q}(0)=\left[\begin{array}{c}
{ }^{\mathrm{g}} \dot{x}_{0}(0) \\
{ }^{\mathrm{g}} \dot{y}_{0}(0) \\
\dot{\theta}(0) \\
\dot{q}_{1}(0) \\
\dot{q}_{2}(0)
\end{array}\right]=\left[\begin{array}{c}
0 \\
0 \\
0 \\
0 \\
0
\end{array}\right] .
$$

Estas condiciones, junto al modelo dinámico Ec. (6) y el control CTC, Ec. (12), permiten el seguimiento de las trayectorias de referencia impuestas. La Tabla I contiene los parámetros dinámicos de los cuerpos que componen el sistema mecánico de estudio, en este caso, el péndulo invertido de 2 GDL. La Ec. (14) permite calcular el punto de momento cero.

El simulador debidamente programado detecta las colisiones de dos vértices de la base con el suelo generando un vector de fuerzas normales y tangenciales que permite observar la rotación y desestabilización de la base, como se ilustrará en la siguiente sección. Los parámetros utilizados en la simulación se describen en la Tabla II.

TABLA I. Parámetros dinámicos del sistema mecánico. La segunda columna corresponde a los momentos de inercia $\left[\mathrm{kg} \cdot \mathrm{m}^{2}\right]$, la tercera contiene las coordenadas del centro de masa multiplicadas por la masa $[\mathrm{kg} \cdot \mathrm{m}]$ y la última columna contiene la masa en $[\mathrm{kg}]$.

\begin{tabular}{cccc}
\hline Cuerpo & $I_{z z}\left[\mathrm{~kg} \cdot \mathrm{m}^{2}\right]$ & $m_{x}[\mathrm{~kg} \cdot \mathrm{m}]$ & $M[\mathrm{~kg}]$ \\
\hline \hline Base & 1.2 & 0.1 & 10 \\
Brazo $q_{1}$ & 1.2 & 0.25 & 1 \\
Brazo $q_{2}$ & 1.2 & 0.25 & 1 \\
\hline
\end{tabular}

TABLA II. Parámetros de Simulación

\begin{tabular}{ccc}
\hline Parámetro & Descripción & Valor \\
\hline \hline$\mu$ & Fricción viscosa & 0.75 \\
$g$ & Gravedad & $9.81 \mathrm{~m} \cdot \mathrm{s}^{-2}$ \\
$2 L$ & Longitud de la base & $0.2 \mathrm{~m}$ \\
$T$ & Paso de simulación & $5 \times 10^{-4} \mathrm{~s}$ \\
$T_{\text {sim }}$ & Tiempo de simulación & $2 \mathrm{~s}$ \\
\hline
\end{tabular}



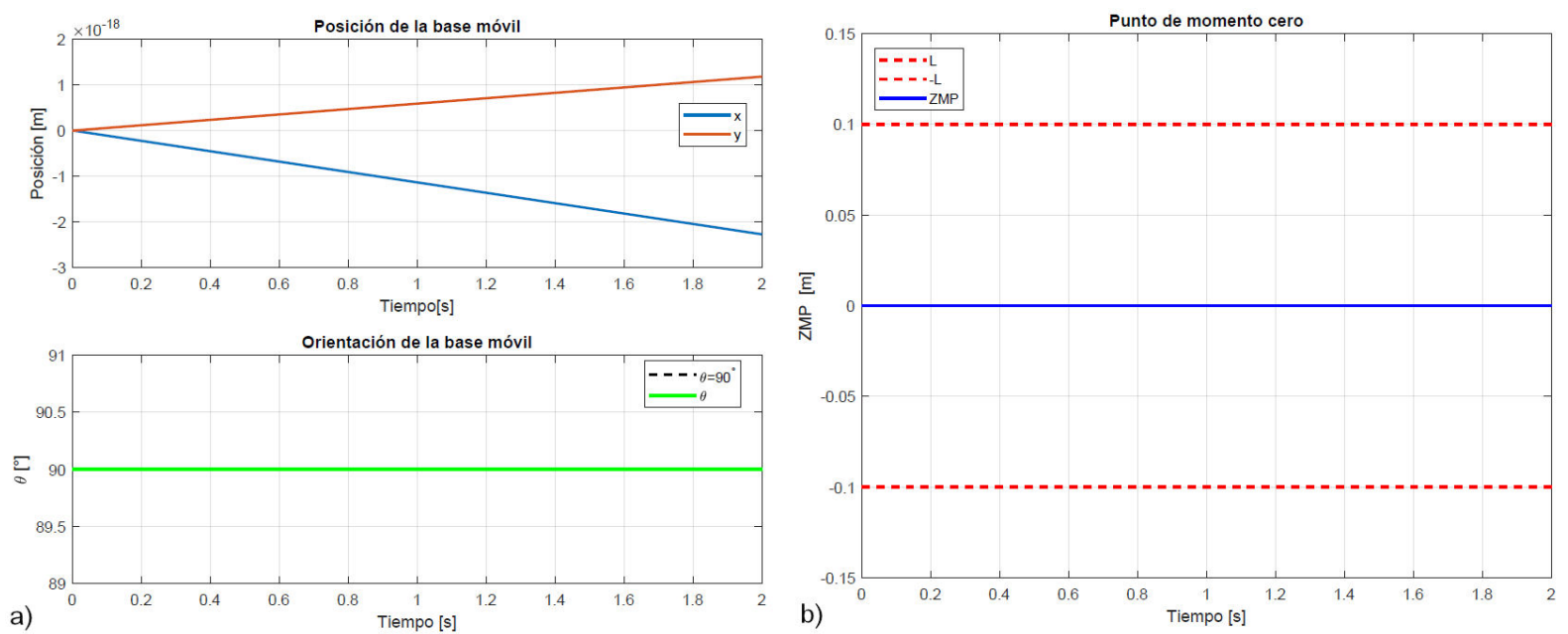

Figura 4. a) Posición y orientación de la base móvil. b) Punto de momento cero (ZMP). Comportamiento dinámico del sistema para las trayectorias estables. Los límites del polígono de sustentación (base) son $[-0.1,0.1] m$ representados por las dos líneas horizontales.
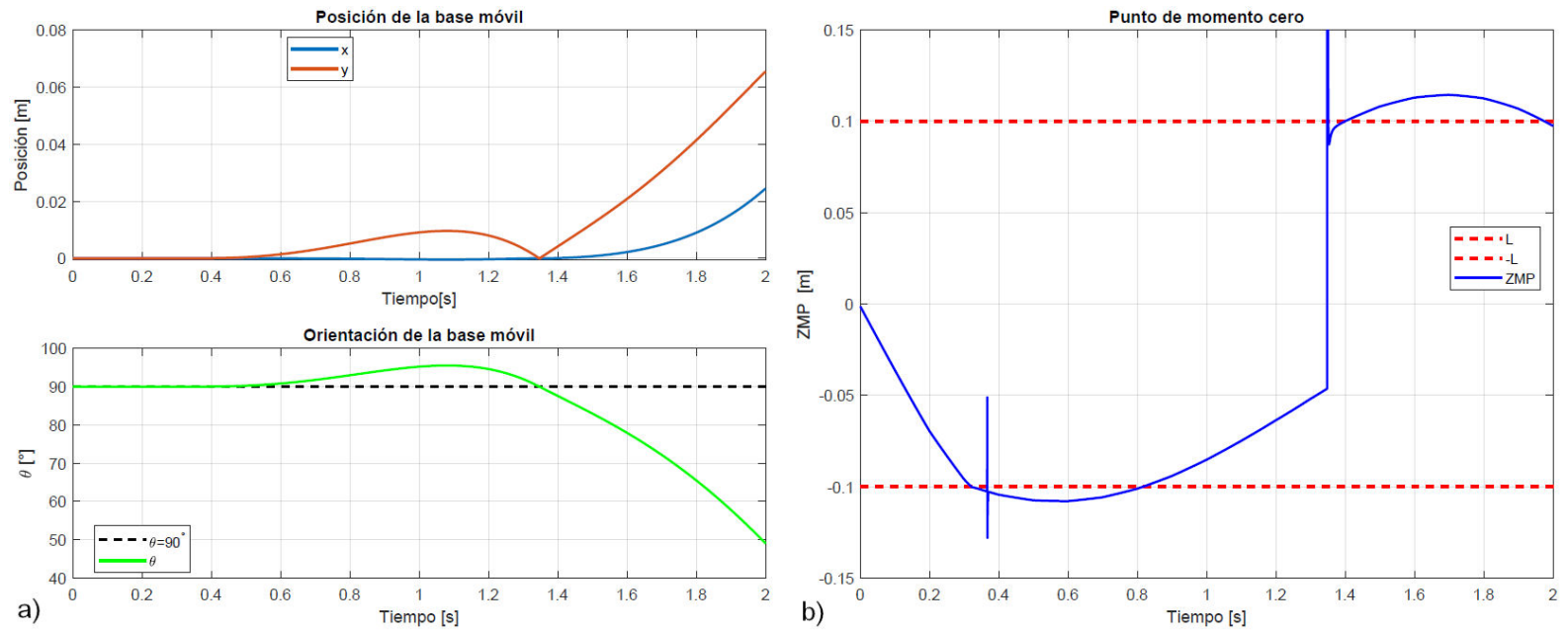

Figura 5. a) Posición y orientación de la base móvil. b) Punto de momento cero (ZMP). Comportamiento dinámico del sistema para las trayectorias inestables. Los límites del polígono de sustentación (base) son $[-0.1,0.1] m$ representados por las dos líneas horizontales.

\subsection{Trayectorias de referencia}

Las primeras trayectorias de referencia son:

$$
\begin{gathered}
q_{1}^{\text {ref }}(t)=0^{\circ} \\
q_{2}(t)^{r e f}=0^{\circ} .
\end{gathered}
$$

Las trayectorias descritas por la Ec. (17) mantendrán el péndulo en posición vertical y el punto de momento cero satisface la condición (Ec. (15)), como se observa en la Fig. 4b), donde $p_{x}=0 \mathrm{~m}$. En este caso, la posición $(x, y)$ de la base y su orientación $\left(\theta=90^{\circ}\right)$ permanecen constantes e iguales a las condiciones iniciales; por tanto, se concluye que estas trayectorias (Ec. (17)) garantizan la estabilidad del sistema dinámico.

Para las trayectorias:

$$
\begin{aligned}
q_{1}^{r e f}(t) & =25^{\circ} \sin 0.9 \pi t \\
q_{2}(t)^{r e f} & =0^{\circ} .
\end{aligned}
$$

Para las trayectorias impuestas en la Ec. (18), el punto de momento cero NO satisface la condición (Ec. (15)). Como se observa en la Fig. 5b), $p_{x}$ no permanece dentro del polígono de sustentación. En el instante $t=0.32 \mathrm{~s}$, el ZMP es menor a $-L$, mientras que en el instante $t=1.37 \mathrm{~s}$ la situación es la opuesta $(L>0.1)$ y la base rota en sentido dextrógiro. En este caso, la posición $(x, y)$ de la base y su orientación $\left(\theta=90^{\circ}\right)$ cambian rápidamente con respecto al tiempo, como lo evidencia la Fig. 5a). Por lo anterior, se concluye que estas trayectorias (Ec. (18)) no permiten la estabilidad del sistema dinámico.

Las Figs. 5a) y 6a) muestran que la estrategia de control CTC permite un seguimiento de las trayectorias de referencia. Sin embargo, el Control CTC no siempre garantiza la estabilidad del sistema mecánico. 

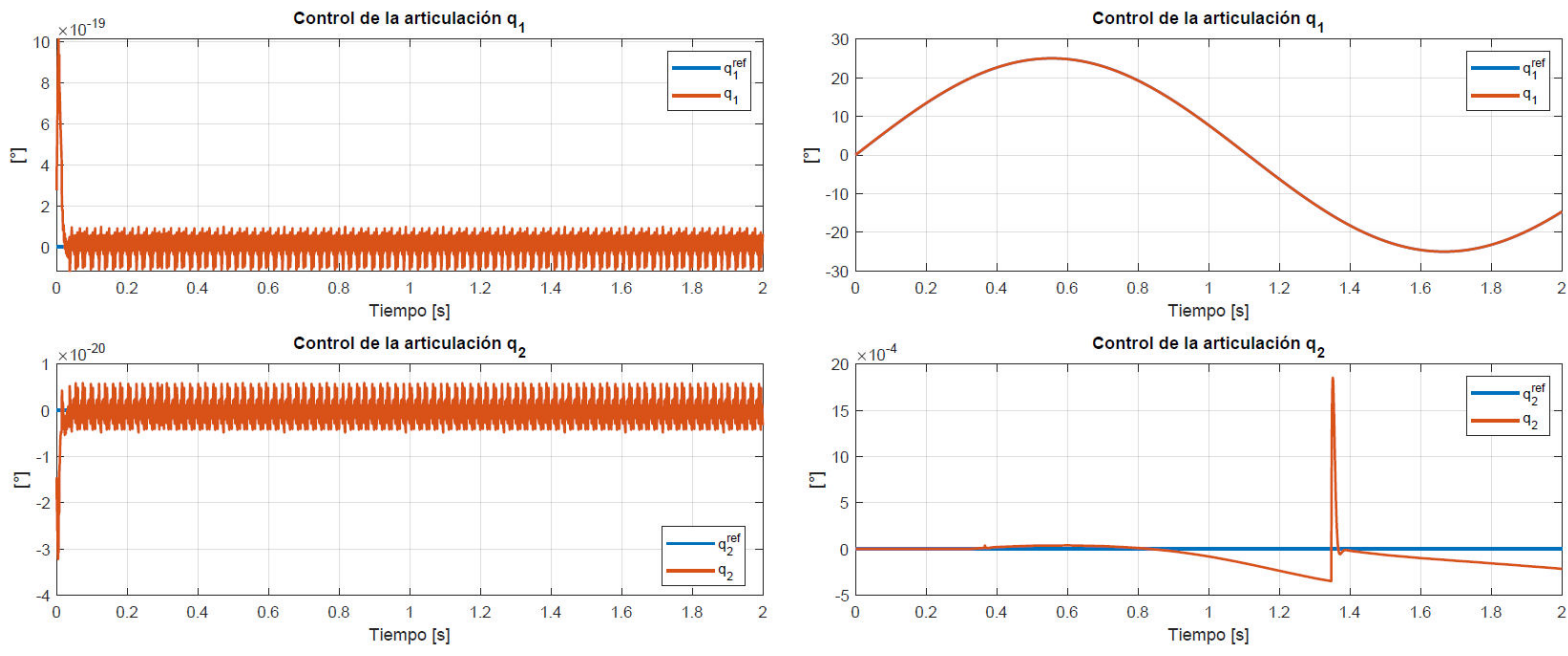

FIGURA 6. a) Seguimiento y control para trayectorias estables. b) Seguimiento y control para trayectorias inestables. Trayectorias de Referencia y Seguimiento para las articulaciones $q_{1}$ y $q_{2}$.

\section{Conclusiones}

En este trabajo se estudió de la estabilidad de un sistema mecánico de base móvil mediante una estrategia de control CTC. Se seleccionó como caso de estudio un péndulo invertido de dos grados de libertad y de base móvil. Las simulaciones muestran que si bien la estrategia de control CTC asegura el seguimiento geométrico de las trayectorias de referencia, no garantiza la estabilidad de la base del sistema.

El estudio de los sistemas mecánicos de base móvil permite evidenciar la diferencia entre limitaciones tecnológicas y limitaciones fundamentales al mostrar que, aunque tecnológicamente es posible diseñar un controlador que permita el seguimiento de trayectorias de referencia, al existir lim- itaciones debido a las propiedades físicas de los sistemas mecánicos (masas y momentos de inercia), el seguimiento de las trayectorias no garantiza la estabilidad de los mismos. Este es un problema abierto a ser resuelto: el seguimiento de trayectorias de referencia en el espacio y tiempo asegurando la estabilidad del sistema dinámico.

\section{Agradecimientos}

Los autores de este artículo expresan sus más sinceros agradecimientos a la Universidad del Cauca por todo el apoyo brindado para la realización del proyecto (Grant number 5411/2020).
1. D. A. Bravo y C. F. Rengifo, Generation from motion capture for a planar biped robot in swing phase, Ingeniería $y$ Ciencia, 11 (2015) 25. https://doi.org/10.17230/ ingciencia.11.22.2

2. J. Tacué, C. Rengifo, and D. Bravo, An experimental energy consumption comparison between trajectories generated by using the cart-table model and an optimization approach for the bioloid robot, International Journal of Advanced Robotic Systems, 17 (2020) 1. https://doi.org/10.1177/ 1729881420917808

3. D. A. Bravo y C. F. Rengifo, Estudio de la dinámica y control de una bicicleta robótica, Rev. Mex. Fís. 17 (2020) 62. https://doi.org/10.31349/RevMexFisE.17.62

4. J. Akesson, A. Blomdell, y R. Braun, Design and control of YAIP.-an inverted pendulum on two wheels robot, in 2006 IEEE Conference on Computer Aided Control System Design, 2006; IEEE International Conference on Control Applications, 2006; IEEE International Symposium on Intelligent Control
(2006) 2178.https : //doi.org/10.1109/CCA.2006. 286204

5. G. V. Raffo, M. G. Ortega, V. Madero, y F. R. Rubio, Twowheeled self-balanced pendulum workspace improvement via underactuated robust nonlinear control, Control Engineering Practice, 44 (2015) 231. https://doi.org/10.1016/ j.conengprac.2015.07.009

6. C. Chevallereau and Y. Aoustin, Optimal reference trajectories for walking and running of a biped robot, Robotica, 19 (2001) 557. https://doi.org/10.1017/ S0263574701003307

7. D. Tlalolini, C. Chevallereau, y Y. Aoustin, Human-like walking: Optimal motion of 8 a bipedal robot with toe-rotation motion, IEEE/ASME Transactions on Mechatronics, 16 (2010) 310. DOI: $10.1109 / \mathrm{TMECH} .2010 .2042458$

8. C. Chevallereau, Time-scaling control for an underactuated biped robot, IEEE Transactions, 19 (2003) 362. DOI: 10. 1109 /TRA.2003.808863 
9. J. Edelmann, M. Haudum, y M. Plochl, Bicycle rider control modelling for path tracking, IFAC-PapersOnLine, 48 (2015) 55. https://doi.org/10.1016/j.ifacol. 2015.05 .070

10. L. Consolini y M. Maggiore, Control of a bicycle using virtual holonomic constraints, Automatica 49 (2013) 2831. https://doi.org/10.1016/j.automatica. 2013.05 .021

11. J. Mauny, M. Porez, y F. Boyer, Symbolic dynamic modelling of locomotion systems with persistent contacts - application to the $3 \mathrm{~d}$ bicycle, IFAC-PapersOnLine, 50 (2017) 7598. https : //doi.org/10.1016/j.ifacol.2017.08.1007

12. N. B. Hung, J. Sung, y O. Lim, A simulation and experimental study of operating performance of an electric bicycle integrated with a semi-automatic transmission, Applied Energy, 221 (2018) 319. https://doi.org/10.1016/j.egypro. 2019.01 .937

13. D. A. Bravo M., C. F. Rengifo R., and J. F. Díaz O., Control of a robotic bicycle, in 2018 IEEE 2nd Colombian Conference on Robotics and Automation (CCRA) (2018), pp. 1.
14. D. A. Bravo, C. F. Rengifo, y J. F. Díaz, Comparative analysis between computed torque control, lqr control and pid control for a robotic bicycle, in 2019 IEEE 4th Colombian Conference on Automatic Control (CCAC) (2019) pp. 1.

15. D. A. Bravo, C. F. Rengifo, y W. Acuña, Dynamics and preview control of a robotics bicycle, in Advances in Automation and Robotics Research. (Springer International Publishing, 2020), pp. 248.

16. D. A. Bravo and C. F. Rengifo, Herramienta para la enseñanza de las ecuaciones de Lagrange basada en la simulacion de sistemas dinámicos, Rev. Mex. Fís. 60 (2014) 111.

17. W. Khalil y E. Dombre, Modeling, Identification and Control of Robots, 3rd ed. USA: (Taylor and Francis, Inc., 2002).

18. M. Vukobratović, Zero-moment point. thirty five years of its life, International Journal of Humanoid Robotics, 01 (2004) 157. https://doi.org/10.1142/ S0219843604000083

19. C. D. Boor, A Practical Guide to Splines. (Springer, 2001). 